Pneumologe 2016 $\cdot 13: 363-364$

DOI 10.1007/s10405-016-0071-0

Online publiziert: 15 . September 2016

๑) Springer-Verlag Berlin Heidelberg 2016

CrossMark

\author{
M. Rose' $\cdot$ O. Karg ${ }^{2}$ \\ 'Fachbereich Medizin, Goethe Universität Frankfurt am Main, Mannheim, Deutschland \\ ${ }^{2}$ Klinik für Intensivmedizin und Langzeitbeatmung, Asklepios Fachkliniken München-Gauting, München, \\ Deutschland
}

\title{
Nichtpharmakologische interventionelle Therapieverfahren in der Pneumologie
}

Liebe Leserinnen und Leser,

das Leitthema dieser Ausgabe der Zeitschrift Der Pneumologe widmet sich nichtpharmakologischen interventionellen Therapieverfahren in der Pneumologie. Wir verknüpfen den Begriff „interventionell“ ja nahezu reflexartig mit der Endoskopie, aber das ist zu eng gedacht. Neben der Endoskopie kommen Interventionen vor allem auch in der Intensiv-, Beatmungs- und Schlafmedizin zum Einsatz. Entsprechend breit ist das Spektrum der Therapien, die in dieser Ausgabe beschrieben werden. Hinzu kommt, dass zwei therapeutische Verfahren mit ihren altersspezifischen Merkmalen sowohl aus der Sicht der Erwachsenenpneumologie, als auch der pädiatrischen Pneumologie dargestellt werden. Es freut uns sehr, dass wir hierfür jeweils zwei Autorengruppen gewinnen konnten.

Natürlich kommt auch die Endoskopie zu Wort: Die Autoren K. Darwiche, St. Eisenmann und W. Gesierich befassen sich mit den Themen bronchiale Thermoplastie und gezielte Lungendenervierung. Die bronchiale Thermoplastie als ultima ratio beim schweren unkontrollierten Asthma bronchiale ist zwar kein ganz neues Verfahren, aber die praktischen Erfahrungen sind im Hinblick auf die seltenen Indikationen immer noch gering und viele Fragen offen. Der Beitrag gibt eine aktuelle Übersicht zur derzeitigen Datenlage.
Ein ganz neues Therapieverfahren für die COPD ist dagegen die gezielte Lungendenervierung (,targeted lung denervation“), d. h. Radiofrequenzablation des Plexus pulmonalis mit dem Ziel einer dauerhaften Vagolyse. Hierzu gibt es bisher noch keine breiten klinischen Erfahrungen, sondern nur sog. Machbarkeitsstudien.

$H$. Teschler und Koautoren beschreiben ausführlich die Methoden der elektrischen Atemwegsstimulation zur Therapie der moderaten bis schweren obstruktiven Schlafapnoe. Es werden u. a. die Unterschiede der verschiedenen Geräte sowie der prädiktive Stellenwert der medikamenteninduzierten Schlafendoskopie im Hinblick auf die Erfolgsrate der Elektrostimulation hervorgehoben und aktuelle Empfehlungen zum klinischen Management zitiert. Der Beitrag ist Pflichtlektüre für jeden Pneumologen, der sein Wissen über Schlafmedizin aktualisieren will.

Der Beitrag von J. Bräunlich und $H$. Wirtz befasst sich mit der nasalen High-Flow-Therapie, d.h. der Zuführung eines angewärmten und angefeuchteten Luft-Sauerstoff-Gemischs über eine spezielle Nasenbrille mit bis zu 60 l/min hohem Fluss. Über dieses Verfahren wird aktuell viel diskutiert, was aber nicht unbedingt zu besserem Verständnis beiträgt. Die Autoren beschreiben ausführlich die bisher bekannten Wirkmechanismen. Die Methode wird derzeit v. a. in der Intensivmedizin bei akuter Hypoxämie eingesetzt. Besonders bei hohen Atemfrequenzen lässt sich eine
Verbesserung der Oxygenierung erreichen. Nach ersten Daten kann die nasale High-Flow-Therapie aber auch eine Hyperkapnie reduzieren. Es bleibt abzuwarten, ob sich hiermit zumindest in einigen Fällen eine Alternative zur nichtinvasiven Beatmung ergibt. Das Verfahren wird somit auch für die Langzeittherapie interessant, möglicherweise als nächster Schritt zu einer individualisierten Atmungsunterstützung.

In der Neonatologie hat die nasale High-Flow-Sauerstofftherapie dagegen bereits einen hohen Stellenwert erreicht und stellt bei bestimmten Indikationen eine Alternative zur CPAP-Atmung dar. Diesem Thema widmet sich der Beitrag von N. Hepping und Koautoren. Sie beschreiben die zu Erwachsenen unterschiedliche Physiologie der Atmung und in Folge dessen die unterschiedlichen Wirkmechanismen von nasalem High-Flow bei Früh- und Neugeborenen. Unterschiede ergeben sich v. a. im Hinblick auf den endexspiratorischen Atemwegsdruck. Die häufigste Indikation für die nasale High-Flow-Therapie ist die Entwöhnung vom Respirator bei Frühgeborenen sowie als weitere Indikationen das Apnoe-Bradykardie-Syndrom und das neonatale Atemnotsyndrom.

Mit dem Thema extrakorporale Membranoxygenierung (ECMO) beim akuten Lungenversagen befasst sich der Beitrag von S. Demirakca und T. Schaible, ebenfalls aus einem neonatologischen Zentrum. Daher bezieht sich der Artikel auch überwiegend auf Neugeborene und Kin- 


\section{Leitthemenübersicht}

der. Die ECMO hat hier bereits einen höheren Stellenwert als bei Erwachsenen, da die Erfolgsraten bei Neugeborenen und Säuglingen am höchsten sind. Wie bei der nasalen High-Flow-Therapie in der Neonatologie handelt es sich um rein intensivmedizinische Indikationen.

Bei Erwachsenen wird aber die venovenöse ECMO bzw. die extrakorporale $\mathrm{CO}_{2}$-Entfernung zunehmend auch beim hyperkapnischen Ventilationsversagen eingesetzt, eine Indikation, die v. a. uns Pneumologen betrifft. Es geht speziell um die Themen Erleichterung einer lungenproduktiven Beatmung und der Beatmungsentwöhnung, Vermeidung einer Intubation beim Versagen einer nichtinvasiven Beatmung und Überbrückung von Wartezeit bis zur Lungentransplantation. Auch hier stehen wir erst am Anfang einer Entwicklung. Diesen Themen widmet sich der Beitrag von P. Lebiedz, S. Kluge und S. Braune.

Allen Autoren herzlichen Dank für ihre geleistete Arbeit. Wir hoffen, dass wir mit diesen Themen eine für Sie spannende Auswahl getroffen haben und wünschen den Lesern viele neue Eindrücke.<smiles>CC1CCCCC1C</smiles>

Prof. Dr. M. Rose

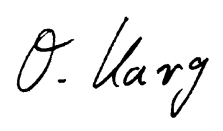

Dr. O. Karg

\section{Korrespondenzadresse}

Prof. Dr. M. Rose
Fachbereich Medizin, Goethe
Universität Frankfurt am Main
Wingerts-Au 64,68259 Mann-
heim, Deutschland
MarkusA_Rose@gmx.de

Interessenkonflikt. M. Rose und O. Karg geben an, dass kein Interessenkonflikt besteht.

Alle Inhalte der Zeitschrift Der Pneumologe finden Sie unter www.springermedizin.de/der-pneumologe

SpringerMedizin.de bietet Ihnen Zugang zu allen elektronisch verfügbaren Ausgaben Ihrer Zeitschrift - unabhängig davon, seit wann Sie die Zeitschrift abonniert haben.

\section{Unser Tipp:}

Mit dem e.Med Kombi-Abo können Sie jederzeit auf alle Inhalte von SpringerMedizin.de zugreifen. AufWunsch erhalten Sie darüber hinaus eine gedruckte Fachzeitschrift Ihrer Wahl.

Testen Sie e.Med 30 Tage lang kostenlos und unverbindlich!

Jetzt informieren unter www.springermedizin.de/eMed oder telefonisch unter 0800-77 80777 (Montag bis Freitag, 10 bis 17 Uhr) 